\section{Sterilization Indicators in Central Sterile Supply Department: Quality Assurance and Cost Implications}

To the Editor-Satisfactory quality control of sterilization processes is of paramount importance in maintaining the reliability of sterile supplies in a Central Sterile Supply Department (CSSD). The CSSD is a department in a healthcare facility that provides sterilized materials to wards, operating rooms, transplant units, and outpatient departments. The materials sterilized by a CSSD include dressing materials, surgical instruments, linens, endoscopes, and other equipment. The sterilizer used depends upon the nature of the item being sterilized, which may be heat resistant or heat sensitive.

The quality control process of each sterilizer varies depending upon the nature of the sterilizer. Herein, we describe our experience with the applications of various sterilization indicators in a CSSD in a 167-bed oncology hospital in eastern India. ${ }^{1,2}$ Our discussion includes cost implications and quality control issues. Physical parameters and chemical and biological indicators are monitored in this center according to the recommendations of the Association for the Advancement of Medical Instrumentation (AAMI) and the International Standards Organization (ISO). ${ }^{3,4}$

The physical monitoring system depends upon sterilization time, temperature, and pressure. In daily work practice, this monitoring can be performed by visual inspection of the gauge-glass in an analog meter, via a program-linked control (PLC system), or with an automatic printout mechanism. The system is calibrated by inserting thermocouples inside the chamber or using a data logger with the manufacturer's predetermined specifications. The data printouts from each sterilizer are documented in the register along with the data for other items sterilized in the same load.

The chemical monitoring system is composed of a set of various indicators based on specific requirements such as equipment monitoring (Bowie-Dick test pack, Class II CI), pack monitoring (internal chemical indicators, Class III-VI), and exposure monitoring (exposure control tape, Class I CI), and others. Every chemical indicator (CI) has 1 stated endpoint value at which a color change occurs. However, Class V CIs must have 3 stated values: at $121^{\circ} \mathrm{C}$, at $135^{\circ} \mathrm{C}$, and at 1 temperature between these values (at which killing of the biological indicator is achieved). Class VI CIs only have 1 stated value for cycle-specific sterilization, depending on the plateau time. All of the chemical indicators are tested using a chemical indicator evaluating resistometer, and each manufacturer follows the ISO 11140-1 standard. ${ }^{3,4}$ A goodquality chemical indicator can easily detect steam quality, non-condensable gases, and proper sterilant penetration inside the sterilizer. Currently, some chemical indicators are also used to check hollow load devices for proper sterilant penetration in a vacuum sterilizer. The air trapped inside the lumen of a hollow device is calculated as lumen diameter multiplied by lumen length, which is called hollow penetration resistance. The shelf life of unused chemical indicators varies with different manufacturers and depends on ambient temperature and humidity level.

The biological monitoring system depends on live nonpathogenic bacterial spores and stringent sterilization requirements. The biological indicators are prepared using a live bacterial spore strip containing a minimum of 1 million live spores. All of the biological indicators are approved by the American Type Culture Collection and are also tested using the biological indicator evaluator resistometer. The D-value of a biological indicator is defined as "the time required in minutes at a certain temperature $\left(121^{\circ} \mathrm{C}\right)$ to reduce the number of viable microorganisms by a factor of 10 ," 5 and the Z-value of a biological indicator is defined as "the number of degrees in Centigrade to reduce the D-value with a factor of $10, " 5$ whereas the sterilization assurance level is the "probability of a single unit being non-sterile after it has been subjected to sterilization." ${ }^{\text {,4 }}$ Finally, the entire set of biological indicators after sterilization is sent to the microbiology department for sterility assurance testing.

With regard to consumables expenditures, the cost implications for the physical monitoring system is negligible and consists of the cost of the thermal paper for printouts. Calibration for physical monitoring should be done each year by an approved external agency.

The cost of chemical indicators is manufacturer based (eg, 3M, USA; Johnson \& Johnson, USA; Gke, Germany) (Table 1). This cost depends on how many critical variables are being measured by the indicators. In our facility, no failures of chemical or biological indicators were recorded in 2013. The total cost of biological indicators was Rs 194,054 (US\$3,234; 16\% of the total indicator expense); this cost is minor compared to the cost of chemical indicators, which was Rs 10,31,616 (US\$17,194; 84\% of total indicator expense). In our center, these costs are supported by the institution as part of the quality-related expenditures of the CSSD. The chemical indicators are used rationally based on the type of materials to be sterilized. For surgical instruments, we use Class V and Class VI indicators, whereas for items such as dressing sets, Class III and Class IV indicators are used. For dressing materials and linens, we use Class I indicators. These classifications are based on Spaulding's classification of criticality of materials with regard to infection control and cost considerations. In our CSSD, the total indicator cost was estimated to account for $6.3 \%$ of the total annual operating cost of the CSSD, and this was approximately $34 \%$ of the total annual consumable cost. These figures have significant implications for CSSDs 
T A B LE 1. Sterilization Indicators in Central Sterile Supply Department at Tata Medical Center

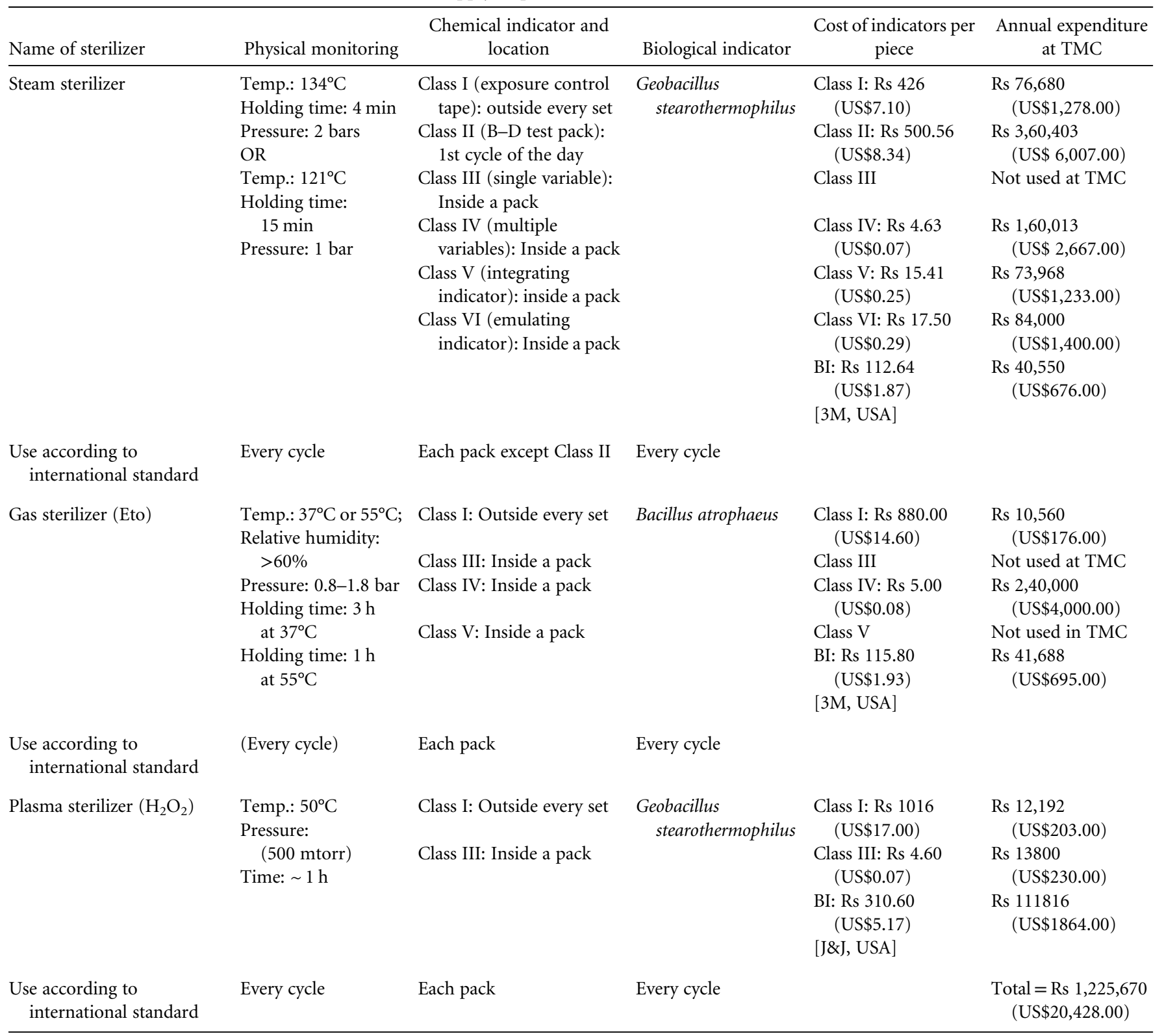

NOTE. TMC, Tata Medical Center; Rs, rupees; BI, biological indicator; J\&J, Johnson \& Johnson. Class I: temperature; Class II: temperature, pressure, time, steam quality, non-condensable gas; Class III: temperature; Class IV: temperature and time; Class V: time, steam, temperature; Class VI: time, steam, temperature (cycle specific).

and hospital administrators. Ensuring quality in sterilization processes requires resources of considerable magnitude. The challenge is to ensure that the sterilization indicators are used efficiently to prevent wastage and maintain quality.
Debabrata Basu, BSc, DHM; Sanjay Bhattacharya, MD, DNB, DipRCPath, FRCPath; Aseem Mahajan, MBBS, MHA; Venkata Raman Ramanan, MS; Mammen Chandy, MD, FRACP, FRCPA

\section{ACKNOWLEDGMENTS}

Financial support. None reported.

Conflict of interest. None reported.
Affiliation: 1. International Safety Center, Apopka, Florida.

Address correspondence to Dr. Sanjay Bhattacharya, MD, DNB, DipRCPath, FRCPath, CCT (UK), Consultant Microbiologist, Tata Medical Center, 
South Lab, 14 Major Arterial Road (E-W), New Town, Rajarhat, Kolkata 700 156, India (drsanjay1970@hotmail.com). Infect Control Hosp Epidemiol 2015;36(4):484-486

(C) 2015 by The Society for Healthcare Epidemiology of America. All rights reserved. 0899-823X/2015/3604-0020. DOI: 10.1017/ice.2014.40

\section{REFERENCES}

1. An overview of central sterile supply department of the Tata Medical Center, Kolkata. In: Scientific Operating Procedures for Sterilization Practices in India. New Delhi: Office of the Principal Scientific Adviser to the Government of India; 2012:165-183.

2. Basu D, Bhattacharya S, Mahajan A, Ramanan VR, Chandy M. The importance of the central sterile supply department in infection prevention and control. Infect Control Hosp Epidemiol 2014;35:1312-1314.

3. Association for the Advancement of Medical Instrumentation. Sterilization of health care products-chemical indicators-Part 1: General requirements. ANSI/AAMI/ISO 11140-1:2005. Arlington (VA): AAMI, 2005. American National Standard.

4. ISO 14161, Sterilization of health care products-biological indicators - guidance for the selection, use and interpretation of results website. https://www.iso.org/obp/ui/\#iso:std:iso:14161: ed-2:v1:en. 2009. Accessed October 20, 2014.

5. Kamolsiripichaiporn S, Subharat S, Udon R, Thongtha P, Nuanualsuwan S. Thermal inactivation of foot-and-mouth disease viruses in suspension. Appl Environ Microbiol 2007;73:7177-7184.

\section{Making the Case for Textiles with a Dual Mechanism of Action}

To the Editor-The paper titled "A Randomized Crossover Trial to Decrease Bacterial Contamination on Hospital Scrubs" by Mallory Boutin et al published in November's issue is an important contribution to the body of evidence needed for the use of technical or engineered textiles as an innovative approach to healthcare-system-based infection prevention. I applaud the authors for making such an important step forward for us as a scientific community as we explore new technologies that hold promise for positive impact not only for patients and healthcare workers but also for public health.

In Ms. Boutin's discussion section, it appears that she and her colleagues have inaccurately interpreted the available published research. She states in her discussion, as it relates to her research, that "(o)ther recent studies testing antimicrobial scrubs but using different products have shown similar ineffectiveness." On the contrary, the study of reference (Bearman $2012^{2}$ ) concluded that the technology they studied was effective.

In short, Bearman et al conducted a randomized clinical trial (RCT) to determine the effectiveness of a breathable, antimicrobial, fluid barrier scrub fabric for reducing the bacterial burden on hands and scrub attire worn by healthcare workers (HCWs) in an intensive care unit (ICU) setting. The technology Bearman et al studied was an active barrier textile, one with a dual mechanism of action of both fluid repellency and antimicrobial attributes. All study participants ( $\mathrm{N}=31$ HCWs) were required to wear an assigned set of scrub attire during a clinical shift. Each HCW underwent unannounced weekly garment and hand cultures. Cultures $(\mathrm{N}=3,324)$ taken at the beginning and end of the shifts included garment cultures taken from the abdominal and leg pockets of the scrub attire.

The researchers found a highly significant statistical $(P=.0002, .0056) 4-7$ mean log reduction in the overall number of methicillin-resistant Staphylococcus aureus (MRSA) CFUs on study scrub attire compared with traditional nonprotective scrub attire worn by HCWs on both the leg and the abdomen. The reduction persisted from the beginning to the end of work shifts.

As Ms. Boutin correctly summarizes, there were no differences in the number of CFUs for vancomycin-resistant Enterococcus (VRE) and Gram-negative rods-not because the study scrubs were not effective, but because the baseline measurements at their facility were too small to measure a statistically significant change. The researchers concluded that "When bundled with known infection prevention strategies such as hand hygiene, antimicrobial impregnated apparel may limit the bacterial burden of the inanimate environment. For settings with high rates of hospital-acquired infections with drug-resistant pathogens such as MRSA, the use of antimicrobial apparel may be a useful adjunct to other infection prevention measures."

It is also important to note that Bearman's findings in the clinical setting were validated in the laboratory findings of Hardwick et al. ${ }^{3}$ Dr. Hardwick and colleagues described the dual mechanism of action of breathable, antimicrobial, fluid barrier fabrics in their published laboratory "Fabric Challenge" test method. Hardwick noted that the combination of an organosilane antimicrobial agent and a hydrophobic barrier chemistry provides an additive effect when combined and results in a higher reduction of MRSA on the fabric than does either the antimicrobial or the fluid barrier alone. The role of the fluid barrier in this dual mechanism is consistent with the CDC/ HICPAC Guideline for Disinfection and Sterilization in Healthcare Facilities (2008), which states that organic matter in the form of serum, blood, pus, or fecal or lubricant material can interfere with the antimicrobial activity of disinfectants. The bioburden reduction results of Hardwick's study strongly correlate with the findings of Bearman and colleagues.

To Ms. Boutin's credit, she is correct regarding textiles with an antimicrobial alone. This was supported in a study conducted by Burden et $\mathrm{al}^{4}$ in which the extent of bacterial contamination of scrub attire and skin were compared when HCWs wore 2 different types of antimicrobial scrub attire compared to traditional nonprotective scrub attire $(\mathrm{N}=105)$. One type of antimicrobial scrub attire was made from a polyester microfiber embedded with an antimicrobial chemical. The second type of antimicrobial scrub attire was made from a polyester/cotton blend 